Portland State University

PDXScholar

Fall 1-1-2015

\title{
Nonlinear Analysis and Digital Pre-Distortion of the SC-FDMA Signals in LTE Uplink System
}

Changwen Zhu

Portland State University

Follow this and additional works at: https://pdxscholar.library.pdx.edu/open_access_etds

Part of the Electrical and Computer Engineering Commons Let us know how access to this document benefits you.

Recommended Citation

Zhu, Changwen, "Nonlinear Analysis and Digital Pre-Distortion of the SC-FDMA Signals in LTE Uplink System" (2015). Dissertations and Theses. Paper 2105.

https://doi.org/10.15760/etd.2103

This Thesis is brought to you for free and open access. It has been accepted for inclusion in Dissertations and Theses by an authorized administrator of PDXScholar. Please contact us if we can make this document more accessible: pdxscholar@pdx.edu. 
Nonlinear Analysis and Digital Pre-Distortion of the SC-FDMA Signals in LTE Uplink System

by

Changwen Zhu

A thesis submitted in partial fulfillment of the requirements for the degree of

\author{
Master of Science \\ in \\ Electrical and Computer Engineering
}

Thesis Committee:

$\mathrm{Fu} \mathrm{Li}$, Chair

Xiaoyu Song

James E. Morris

\author{
Portland State University \\ 2014
}




\begin{abstract}
Single-carrier frequency division multiple access (SC-FDMA) has become a popular alternative to orthogonal frequency division multiple access (OFDM) in multi-user communication on LTE uplink systems. This is primarily due to the low peak-toaverage power ratio (PAPR) of SC-FDMA compared to that of OFDM. Long-term evolution (LTE) uses SC-FDMA on the uplink to exploit this PAPR advantage to reduce transmit power amplifier (PA) back-off in mobile user terminals. However, the latest generation of communication systems requires high power efficiency and a large quantity of capacity in transmitting mobile data, bringing out some other critical problems: 1) the nonlinearity of Radio Frequency (RF) power amplifiers inevitably affects the power efficiency. Working beyond the saturation point is the main reason for power amplifiers having nonlinear properties; 2) In order to obtain adequate capacity, wide bandwidth is applied to the latest communication systems. Since in previous systems the pre-distorter would focus on memory-less distortion with relatively narrow bandwidth, this change makes memory distortion become a serious issue, degrading the transmission quality in the wireless communication systems.

The intent of this thesis is to present nonlinear analysis of the SC-FDMA Spectrum with the RF power amplifier. Relevant mathematical models were considered and applied to the RF power amplifier in terms of intermodulation products and the thirdorder intercept point. The equivalent mathematical model is applied for the first time to SC-FDMA signals and with the previous formulation of the PA model, the derivation of the expressions for spectrum regrowth of amplified SC-FDMA signals
\end{abstract}


was first established and finally simulated with Matlab software. The digital predistortion (DPD) technology was also applied to SC-FDMA signals for the first time in this thesis. An inverse intermodulation and Autoregressive Moving-Average (IMARMA) model was introduced to linearize the PA distortion with memory in the LTE uplink system. The DPD was finally implemented by Matlab R2010b.

Conclusions are drawn that amplified power emission levels can be expressed by the form of third-order intercept point $\left(\mathrm{IP}_{3}\right)$. The expressions for spectrum regrowth of amplified SC-FDMA signals have been verified for the first time with the comparison of simulation, measurement and calculation results. The effects of third order intermodulation have a greater impact than higher order components with respect to out-of-band emission power levels. Furthermore, the DPD algorithm reduced the spectrum regrowth of SC-FDMA signals by $12 \mathrm{~dB}$. The proposed pre-distorter can effectively solve the distortion problem caused by the memory effect in RF power amplifier. 


\section{Acknowledgements}

I feel so fortunate to have all kinds of support from many helpful people. I would like to express the deepest appreciation to my advisor and thesis committee chair, Professor $\mathrm{Fu} \mathrm{Li}$, for his constant support, encouragement, enthusiasm, and patience in guiding this research. He introduced me to the various aspects of wireless communication systems, and helped me to find this exciting research topic. Without his invaluable probing comments and insightful suggestions this thesis would not have been possible.

I owe special thanks to Professor Xiaoyu Song and Professors James Morris, for their guidance and support as the members of the thesis committee. My appreciation should also be given to Xiao Li, the Ph.D student in our video image processing group, who provided me the academic quest during the whole time.

My special thanks to my friends in Portland: Ming Shen, Qi Li, Siyuan Yan, Bosi Chen and Peng Gao. My special thanks also to my friends in the US, UK and China: Boyang Lu, Linqing Luo, Danjun Xu, Dapeng Yuan, Xuanzi Xia, Yujing Zhang, Yunwei Wu and Dan Wu.

This thesis is dedicated to my parents Bingguo Zhu and Min Jin, and my grandparents, for their constant and unconditional support and love. They are my first mentors who nurtured my growth and instilled in me righteous values for living. 


\section{Table of Contents}

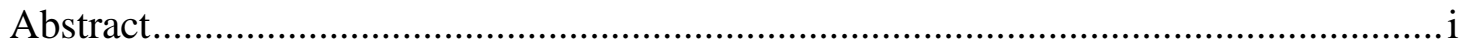

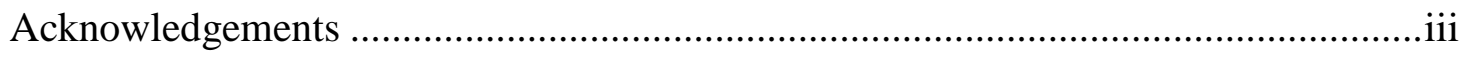

List of Tables ............................................................................................................. vi

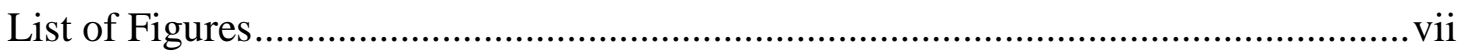

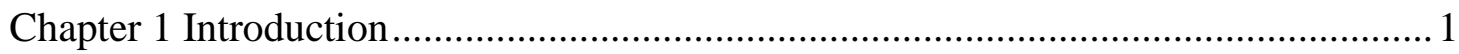

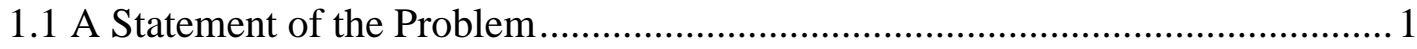

1.2 Review of Literature ............................................................................... 2

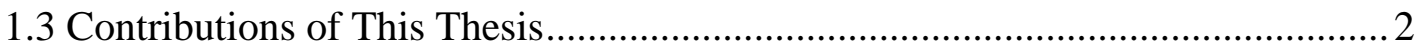

1.3.1 Nonlinear Analysis of SC-FDMA Spectrum .......................................... 2

1.3.2 The Digital Pre-Distortion of SC-FDMA Spectrum ................................... 3

Chapter 2 Nonlinear Analysis of SC-FDMA Spectrum for LTE Up-link.................... 4

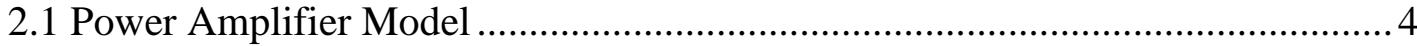

2.1.1 Nonlinearity of Power Amplifiers ............................................................ 4

2.1.2 Traditional Memory-less Model ............................................................. 5

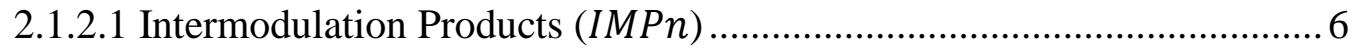

2.1.2.2 Third-order Intercept Point (IP3) ................................................ 8

2.1.2.3 Fifth-order Intercept Point (IP5) ................................................... 10

2.1.3 RF Power Amplifier's Mathematical Model............................................. 11

2.1.3.1 Mathematical Model of with IP3 only ........................................... 11

2.1.3.2 Mathematical Model of with IP3 and IP5 ..................................... 12

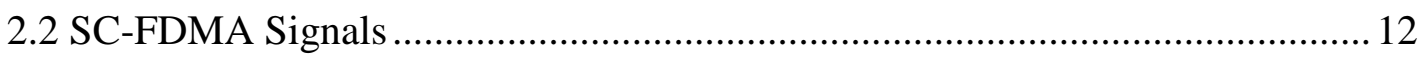

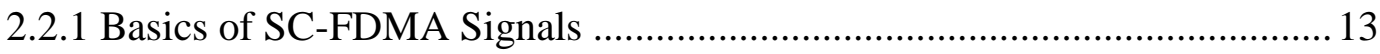

2.2.2 The Equivalent Mathematical Model of SC-FDMA signals...................... 14

2.3 Spectrum Regrowth of Amplified LTE Uplink Signals ................................... 16

Chapter 3 The Digital Pre-Distortion of the SC-FDMA Signals in LTE Up-link

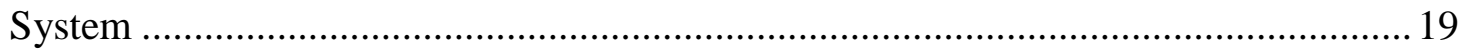

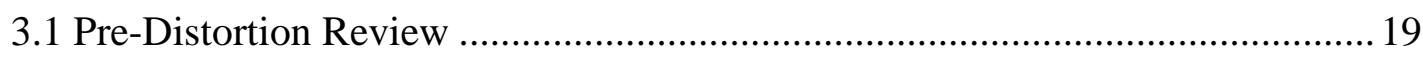

3.2 Spectrum Regrowth Analysis with Pre-Distortion ....................................... 20 
3.2.1 Block A: The Nonlinear Model............................................................ 21

3.2.2 Block C: The Memory Effect Model ...................................................... 21

3.2.3 Block B: The Inverse Polynomial of PA................................................. 23

3.2.4 Block D: The Inverse Memory Effect of PA ........................................... 23

Chapter 4 Analysis and Experimental Study of SC-FDMA Signals ........................... 25

4.1 SC-FDMA Spectrum for LTE Up-link System .......................................... 25

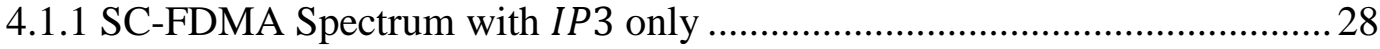

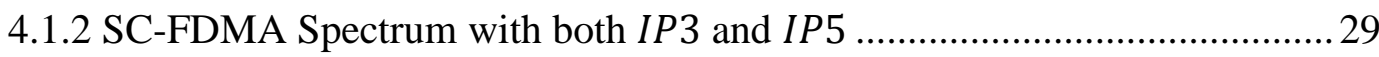

4.2 The Digital Pre-Distortion of the SC-FDMA Signals .................................... 30

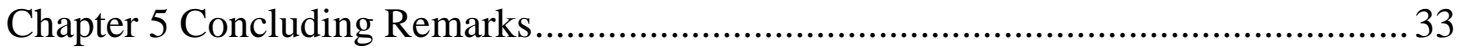

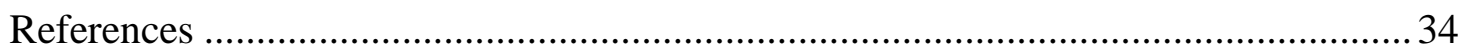




\section{List of Tables}

Table 2.1 Intermodulation distortion products in a Two-Tone System...................... 7

Table 2.2 Parameters applied in two-tone system .............................................. 7

Table 2.3 Parameters applied in the simulation math model................................... 15

Table 4.1 Experimental Instruments.............................................................. 27 


\section{List of Figures}

Figure 2.1 Characteristics of ideal amplifiers............................................................. 4

Figure 2.2 Characteristics of practical amplifier ..................................................... 5

Figure 2.3 Spectrum of Products in the two-tone system............................................ 8

Figure 2.4 The definition of third-order intercept point ............................................... 9

Figure 2.5 Two-tone test for the third-order intermodulation ........................................ 10

Figure 2.6 Two-tone test for the fifth-order intermodulation ........................................ 11

Figure 2.7 Transmitter and receiver of OFDM \& SC-FDMA systems ........................... 13

Figure 2.8 CCDF of PAPR of OFDM and SC-FDMA signals ..................................... 14

Figure 2.9 Power spectrum comparison among simulation, measurement and

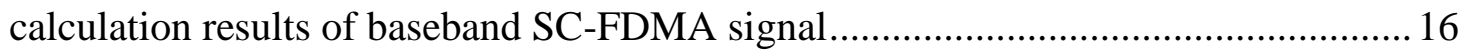

Figure 3.1 The Structure of DPD process................................................................ 20

Figure 4.1 The flow chat of simulation process …………………................................ 26

Figure 4.2 Experimental setup of spectrum measurement of SC-FDMA signals ........27

Figure 4.3 Power spectrum comparison among amplified signals with IP3 only in up-

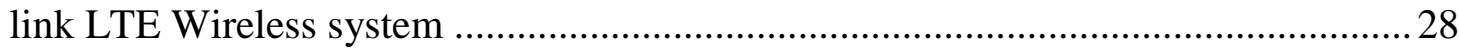

Figure 4.4 Power spectrum comparison with both IP3 and IP5 …..............................30

Figure 4.5 The power spectrum comparison of SC-FDMA signals .............................. 31 


\section{Chapter 1 Introduction}

\subsection{A Statement of the Problem}

The nonlinearity of RF power amplifiers is one of the main concerns in wireless communication systems. The amplifier won't have a non-linear problem before it attains the saturation point. However, as LTE advanced systems require greater capacity and reliability in transmitting mobile data, the linear region of the amplifier can't satisfy the ever-increasing requirement due to the lack of power efficiency. In this situation, the power amplifier is always designed to operate beyond the saturation point, which will result in nonlinearity of the amplifier. The nonlinear distortion is one of the principal reasons for degrading the transmission quality in the wireless communication systems. SC-FDMA is commonly used in the uplink of LTE and LTE advanced systems because of the lower PAPR compared with the widely used OFDM scheme. However, the nonlinearity caused by the characteristic of the RF power amplifiers is still a critical problem, and there is little research in linearization of SCFDMA signals with RF power amplifiers in LTE uplink systems.

Digital pre-distortion (DPD) is generally applied by employing digital baseband distortion ahead of the amplifier to compensate for the nonlinearity effects and to provide efficiency enhancements for high power amplifiers in the different communication systems. The added distortion has totally opposite nonlinear features from the nonlinearity of the amplifier. With the inclusion of intermodulation products (IMPs) in the DPD algorithm, the spectrum regrowth of the amplified signals can be counteracted well in terms of memory-less PA models. The latest generation wireless 
communication systems, like LTE and LTE advanced systems, require larger bandwidth, whereas the additional memory effect reduces precision of the traditional pre-distorter.

\subsection{Review of Literature}

Previously the research group in Portland State University has already conducted indepth studies of the power amplifier nonlinearity and digital pre-distortion. Heng Xiao built the mathematical relationship between $3^{\text {rd }}$ and $5^{\text {th }}$ order coefficients and intercept points of RF power amplifiers [1]. Chunming Liu analyzed nonlinearity of RF power amplifiers with OFDM signals [2]. Bosi Chen extended that knowledge to LTE and WiMAX systems [3] and Xiao Li established the model of high order spectrum regrowth of OFDM signals in $4 \mathrm{G}$ communication systems and proceeded to predistortion with memory effects [4]. Furthermore, the impact of the power amplifier on SC-FDMA signals has been discussed in recent studies [5].

\subsection{Contributions of This Thesis}

\subsubsection{Nonlinear Analysis of SC-FDMA Spectrum}

In this thesis, the nonlinearity of SC-FDMA signals has been analyzed and predicted in spectral terms. Owing to the nonlinearity of RF power amplifiers, an analytic model is introduced on the basis of the third-order intercept point $\left(I P_{3}\right)$ since this could be considered as a weakly nonlinear system [6]. The model is subsequently applied for 
the first time to SC-FDMA signals for the nonlinear analysis of spectrum regrowth. Finally the simulation results have been compared with measurement.

\subsubsection{The Digital Pre-Distortion of SC-FDMA Spectrum}

Digital Pre-Distortion (DPD) based on the efficient and flexible inverse intermodulation and Autoregressive Moving-Average (IM-ARMA) model is applied to SC-FDMA signals in the LTE uplink system to linearize the PA distortion with memory. It captures the nonlinearity effect and the memory effect first with two separate models based on the Hammerstein structure, and then applies the inverse polynomial model and the inverse IM-ARMA model to compensate for the two effects respectively. The improvement of the DPD algorithm for the SC-FDMA signals has been verified with a series of experimental measurements for the first time in this thesis. 


\section{Chapter 2 Nonlinear Analysis of SC-FDMA Spectrum for LTE Up-link}

\subsection{Power Amplifier Model}

\subsubsection{Nonlinearity of Power Amplifiers}

The nonlinearity of RF power amplifiers is one of the principal reasons for degradation of the transmission quality in wireless communication systems. Figures 2.1 and 2.2 show that practical amplifiers perform different from the ideal [7]. The practical amplifier (Figure 2.2) performs as the same as the ideal one (Figure 2.1) only in the linear region, while in both the cut-off and saturation regions the output suffers from the nonlinearity of the amplifier [8].

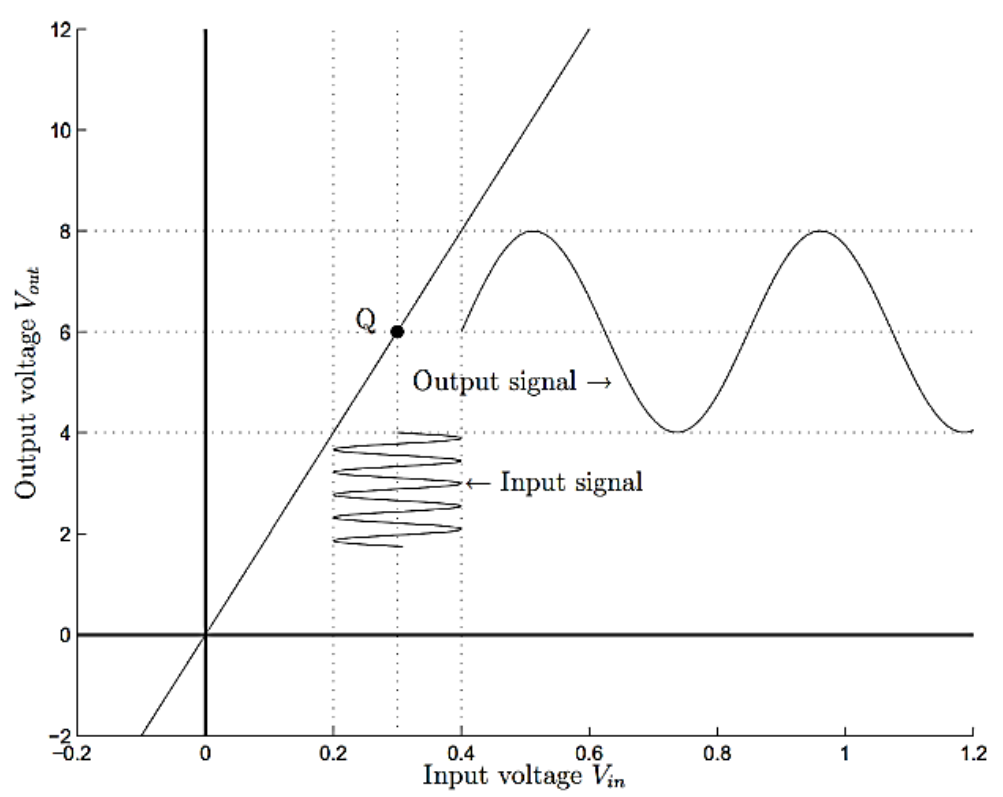

Figure 2.1 Characteristics of ideal amplifiers 


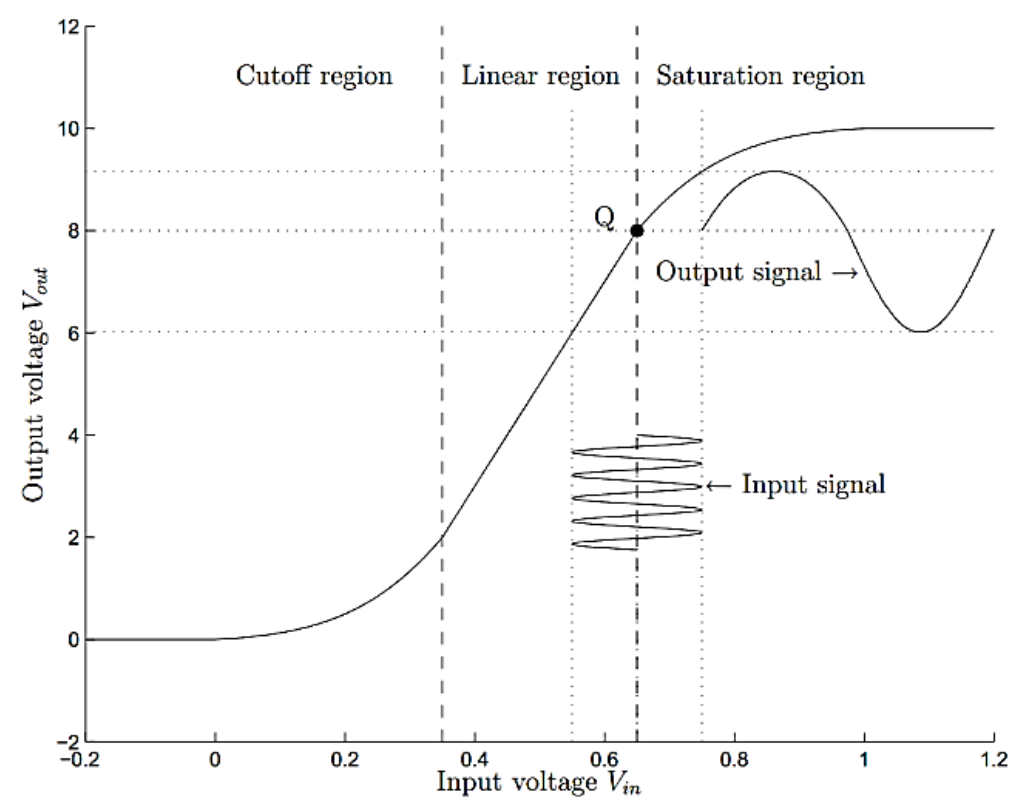

Figure 2.2 Characteristics of practical amplifier

\subsubsection{Traditional Memory-less Model}

For an ideal power amplifier, the output performs perfectly linearly, according to scaling and superposition principles, and is described as

$$
V_{\text {out }}(t)=G V_{\text {in }}(t)
$$

where output $V_{\text {out }}(t)$ is the product of the input $V_{\text {in }}(t)$ and a scalar coefficient $G$ which represents the amplifier gain.

However, for a practical amplifier, as shown in Figure 2.2, the output will saturate to the maximum output level even if the input continues to rise. Therefore, the Taylor series [9] is applied in this case

$$
\begin{aligned}
V_{\text {out }}(t) & =G\left[V_{\text {in }}(t)\right]=\sum_{i=0}^{\infty} G_{n} V_{\text {in }}{ }^{n}(t) \\
& =G_{0}+G_{1} V_{\text {in }}(t)+G_{2} V_{\text {in }}{ }^{2}(t)+G_{3} V_{\text {in }}{ }^{3}(t)+\cdots G_{n} V_{\text {in }}{ }^{n}(t)
\end{aligned}
$$


where $G_{0}$ is the DC output offset, and $G_{n}$ is the coefficient corresponding to the term $V_{\text {in }}{ }^{n}(t) . G_{1} V_{\text {in }}(t)$ is the first order term which is the linear part similar to the ideal amplifier. However, the other terms, such as the second order term $G_{2} V_{i n}{ }^{2}(t)$, cause nonlinear distortion if not all coefficient $G_{n}(n>1)$ are zero. Hence, as $G_{1}$ must always be much larger than $G_{n}(n>1)$, in order to perform as an acceptable linear amplifier.

\subsubsection{Intermodulation Products $\left(I M P_{n}\right)$}

Intermodulation distortion is widely used to measure the linearity of RF components, especially power amplifiers [10]. It often occurs when frequencies other than the input frequencies are generated by the nonlinear features of amplifiers. These are referred to as intermodulation products called $I M P_{n}$, where ' $n$ ' represents the order of the intermodulation products. $I M P_{n}$ is a result of an interaction between two signals in an electronic apparatus.

In a Two-Tone System, the input signal with two frequencies $\omega_{1}$ and $\omega_{2}$ is described as

$$
V_{\text {in }}(t)=A_{1} \cos \left(\omega_{1} t\right)+A_{2} \cos \left(\omega_{2} t\right)
$$

Intermodulation products at the frequencies indicated in Table 2.1 are close to the input signals and are difficult to filter out. 


\begin{tabular}{|c|c|}
\hline & Frequency \\
\hline Fifth-order & $3 \omega_{1}-2 \omega_{2}$ \\
\hline Third-order & $2 \omega_{1}-\omega_{2}$ \\
\hline First Signal & $\omega_{1}$ \\
\hline Second Signal & $\omega_{2}$ \\
\hline Third-order & $2 \omega_{2}-\omega_{1}$ \\
\hline Fifth-order & $3 \omega_{2}-2 \omega_{1}$ \\
\hline
\end{tabular}

Table 2.1 Intermodulation distortion products in a Two-Tone System

For a bandpass system, the two frequencies $\omega_{1}$ and $\omega_{2}$ would be relatively similar $\left(\omega_{1} \approx \omega_{2} \gg 0\right)$. In Figure 2.3, the input frequencies are $0.9985 \mathrm{GHz}$ and $1.0015 \mathrm{GHz}$ so the spacing between the two adjacent tones is $3 \mathrm{MHz}$. Additional parameters are shown in Table 2.2.

\begin{tabular}{|c|c|}
\hline Vertical division & $10 \mathrm{~dB}$ \\
\hline Fundamental & $-9.14 \mathrm{dBm}$ \\
\hline Center frequency & $1.0 \mathrm{GHz}$ \\
\hline Span & $32.0 \mathrm{MHz}$ \\
\hline
\end{tabular}

Table 2.2 Parameters applied in two-tone system

The spectrum was measured and recorded in Figure 2.3, it could be concluded that these third-order terms are always the largest of all intermodulation products. Also since they are close to the original signals and hard to filter, they are always 
considered as the principal concern for filtering the interference out of the desired signal.

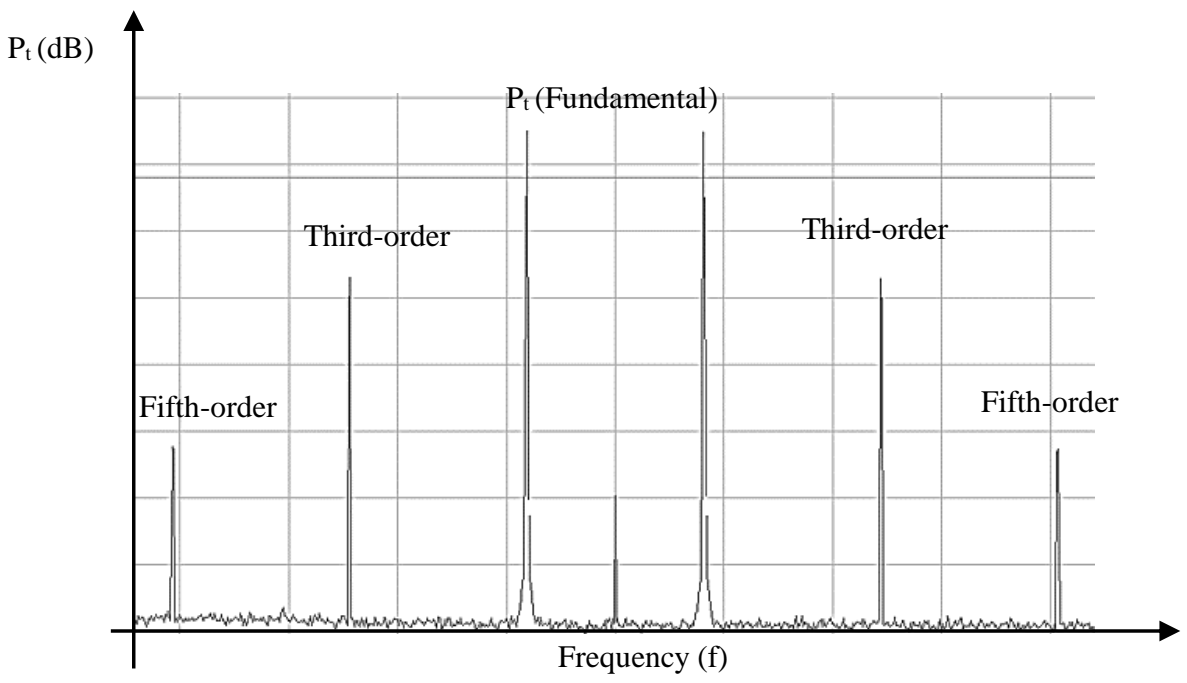

Figure 2.3 Spectrum of Products in the two-tone system

\subsubsection{Third-order Intercept Point $\left(I P_{3}\right)$}

The third-order intercept point $\left(I P_{3}\right)$ is an imaginary point in theory, where the thirdorder intermodulation distortion signal has the same amplitude as the output signals, shown in Figure 2.4 [8]. The third-order intercept point is regarded as an effective measuring method for weakly nonlinear systems. With applying a low-order polynomial, inferred from the Taylor series expansion, the third-order intercept point helps build a meaningful model of linear amplified signals including third-order nonlinear products. 


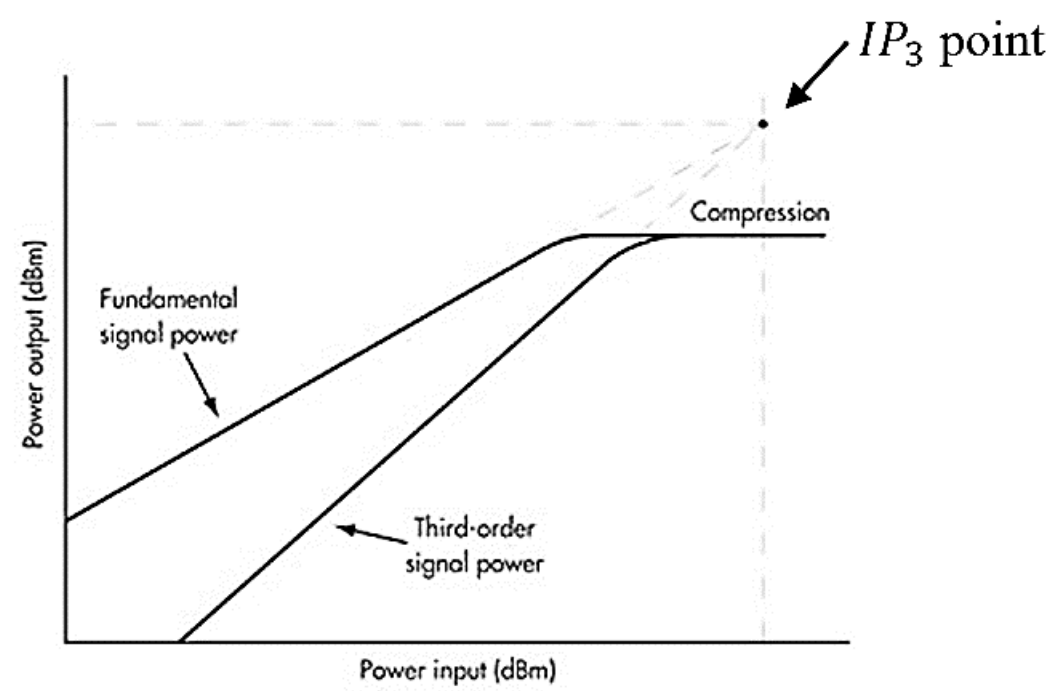

Figure 2.4 The definition of third-order intercept point

A higher power output at the intercept point corresponds to better linearity and lower IMD [8]. Hence the value of $I P_{3}$ becomes important when evaluating the operational range of the amplifier, which relates to the maximum signal power without IMD occurring. To measure the level of co-channel interference using intercept points, a two-tone test is applied to measure the model expression of $I P_{3}$, which is written as [1]

$$
I P_{3}=P_{t}+\frac{I M_{3}}{2}
$$

where $P_{t}$ is the power of the original output tone signal and $I M_{3}$ is the difference between $P_{t}$ and the $3^{\text {rd }}$ order intermodulation output power, shown in Figure 2.5 [2]. 


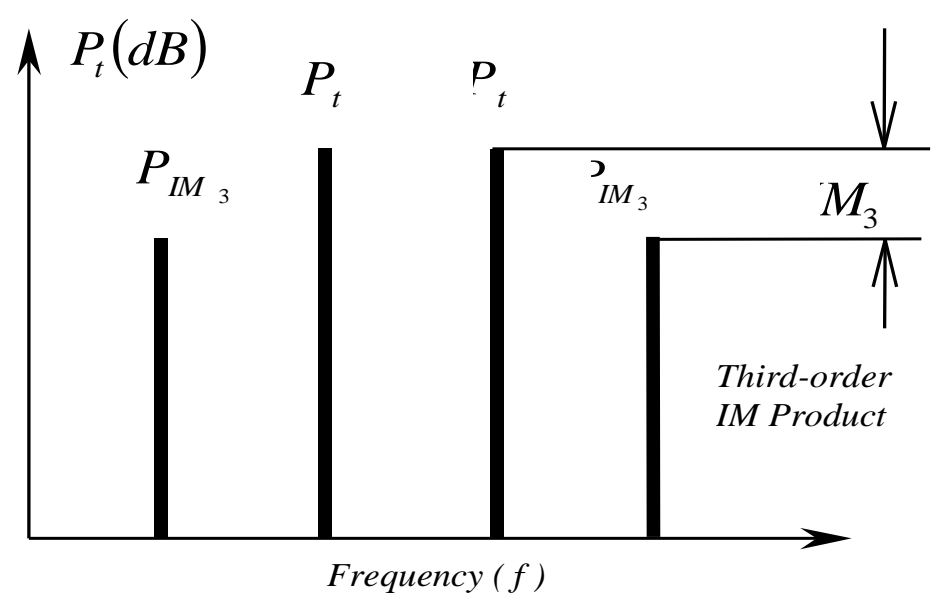

Figure 2.5 Two-tone test for the third-order intermodulation

\subsubsection{Fifth-order Intercept Point $\left(I P_{5}\right)$}

The fifth-order Intercept Point $\left(I P_{5}\right)$ is defined as the point where the fundamental and $I M_{5}$ response intersect [11]. The nonlinearity of the power amplifier is always described and determined by $I P_{3}$ provided by the manufacturer's datasheets. However, the spectrum could gain increased precision by including both $I P_{3}$ and $I P_{5}$. Since $I P_{5}$ is not given by the manufacturer's datasheets, a two-tone test is used, as for $I P_{3}$, to measure the model expression of $I P_{5}$, which is written as [2]

$$
I P_{5}=P_{t}+\frac{I M_{5}}{4}
$$

where $I M_{5}$ is the difference between $P_{t}$ and the $5^{\text {th }}$ order intermodulation output power, shown in Figure 2.6 [2]. 


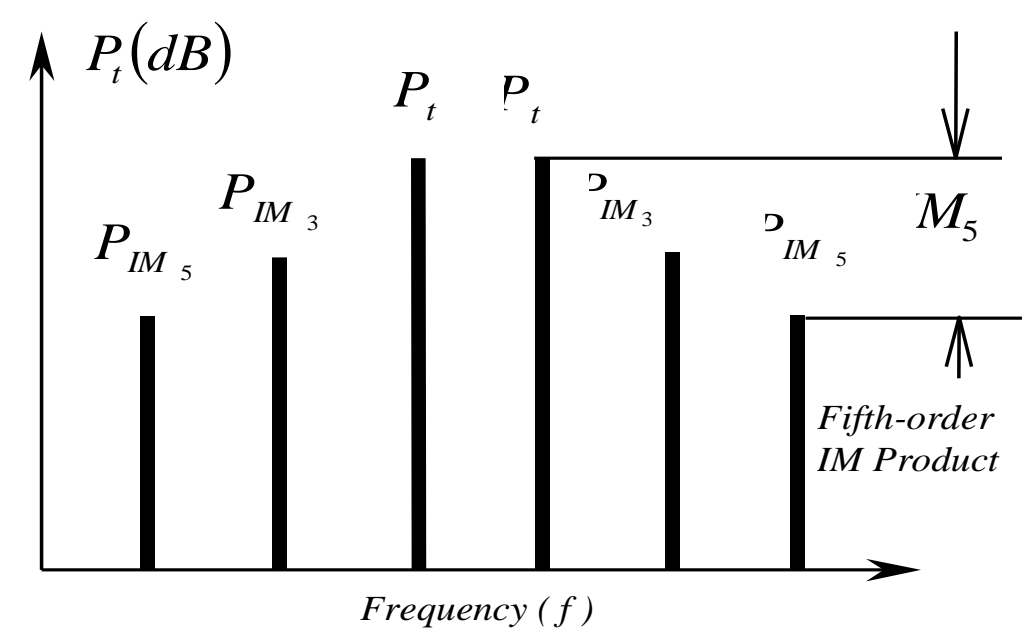

Figure 2.6 Two-tone test for the fifth-order intermodulation

\subsubsection{RF Power Amplifier's Mathematical Model}

\subsubsection{Mathematical Model of with $I P_{3}$ only}

Owing to the nonlinearity of RF power amplifiers, the Taylor model is established on the basis of the intercept points $\left(I P_{n}\right)$, and is fitted for weakly nonlinear systems and devices to:

$$
y(t)=\sum_{i=0}^{\infty} a_{2 \mathrm{i}+1} s^{2 i+1}(t)
$$

where $y(t)$ is the output and $\mathrm{s}(t)$ is input signal of the power amplifier in the passband [12]. Because even-order terms, which are more than one carrier frequency away from the centre frequency, can be easily filtered out[1], only the third order intercept point is considered for the analytical model in this section, expressed as

$$
\mathrm{y}(t)=a_{1} \mathrm{~s}(t)+a_{3} \mathrm{~s}^{3}(t)
$$


The coefficients $a_{1}$ and $a_{3}$ depend on the linear gain $G$ of the amplifier and $I P_{3}$, both of which are always introduced in datasheets from the manufacturers of RF power amplifiers. For the amplifier with gain compression $\left(a_{3}<0\right)$,

$$
a_{1}=10^{\frac{G}{20}}, a_{3}=-\frac{2}{3} 10^{\left(-\frac{I P_{3}}{10}+\frac{3 G}{20}\right)}
$$

\subsubsection{Mathematical Model of with $I P_{3}$ and $I P_{5}$}

In this section, both $I P_{3}$ and $I P_{5}$ are considered for the analytical model, expressed as

$$
y(t)=a_{1} s(t)+a_{3} s^{3}(t)+a_{5} s^{5}(t)
$$

The extra coefficient $a_{5}$ depends on the linear gain $G$ and $I P_{5}$. For the amplifier with gain compression,

$$
a_{5}=-\frac{2}{5} 10^{\left(-\frac{I P_{5}}{5}+\frac{G}{4}\right)}
$$

\subsection{SC-FDMA Signals}

Single-Carrier Frequency-Division Multiple Access (SC-FDMA) is widely used in the up-link of long-term evolution (LTE) and LTE-Advanced systems. It is similar to Orthogonal Frequency-Division Multiplexing (OFDM) used in the downlink of LTE systems [13], but with an extra Discrete Fourier Transform (DFT) block prior to subcarrier mapping, SC-FDMA shows its strength in a robust high peak-to-averagepower ratio (PAPR) which produces benefits of both terminal expense and power efficiency [14]. However, the nonlinearity caused by the characteristic of the RF power amplifiers is still a critical problem, and there is very little research into linearization for the SC-FDMA signals. 


\subsubsection{Basics of SC-FDMA Signals}

SC-FDMA with cyclic prefix (CP) is widely applied in the up-link of long-term evolution (LTE) systems due to its salient advantage of low peak-to-average-power ratio (PAPR) contributing to a low terminal expense and high power efficiency of the transmitter [14]. It has some similarities to Orthogonal Frequency-Division Multiplexing (OFDM) used in the down-link of LTE systems, but the unique characteristic of SC-FDMA is embodied in different carrier transmission schemes, prompting SC-FDMA to be a more valuable alternative to OFDM. A SC-FDMA transceiver occupies an additional Discrete Fourier Transform (DFT) block prior to subcarrier mapping, illustrated in Figure 2.7 [15].

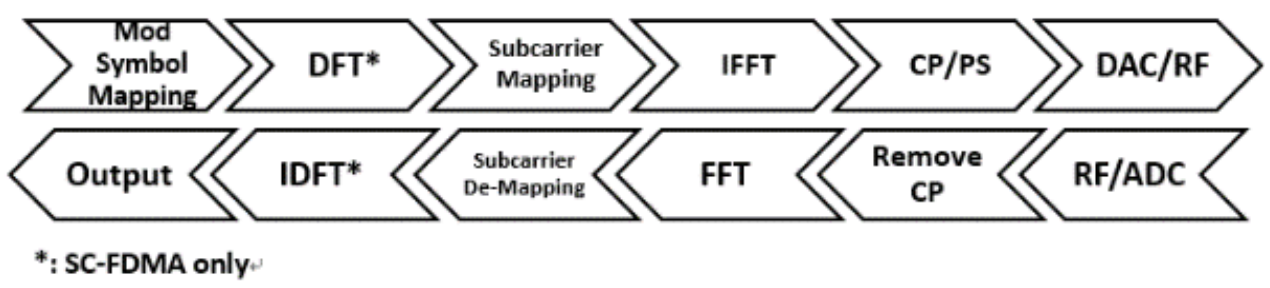

Figure 2.7 Transmitter and receiver of OFDM \& SC-FDMA systems

In a transmitter, data symbols are transformed from the time domain to the frequency domain. After subcarrier mapping, the Inverse Discrete Fourier Transform (IDFT) operation performs the same feature with a single carrier signal with lower power variations. Moreover, the transmitted signals are operated in the frequency domain through shifting the IDFT inputs, in order to satisfy requests for flexible bandwidth [16]. By contrast, because of the increasing number of subcarriers, outputs in the time 
domain for OFDM signals behave like Gaussian noise performing high PAPR values compared with SC-FDMA signals in Figure 2.8 [17]. On the vertical axis, the value represents the probability that the PAPR value is larger than the $\mathrm{PAPR}_{0}$ value.

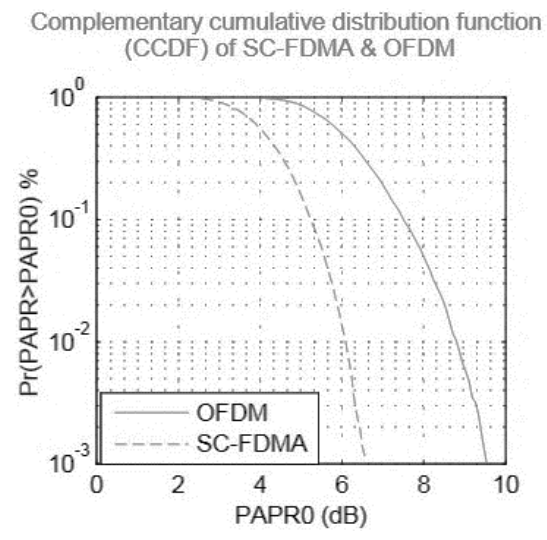

Figure 2.8 CCDF of PAPR of OFDM and SC-FDMA signals

\subsubsection{The Equivalent Mathematical Model of SC-FDMA signals}

The nonlinearity of SC-FDMA signals is analysed and predicted with respect to the spectrum.

The analytical model of a SC-FDMA symbol is expressed as [18]

$$
b(t)=w_{T}(t) \sum_{k=-N_{S T} / 2}^{N_{S T} / 2} a_{k, n} e^{j 2 \pi k \Delta f\left(t-T_{G I}\right)}
$$

A SC-FDMA symbol consists of a set of modulated data named as $a_{k, n}$ in expression (2.11) showing the data in the $\mathrm{n}^{\text {th }} \mathrm{SC}-$ FDMA symbol and the $\mathrm{k}^{\text {th }}$ subcarrier. In the SCFDMA system, a time-window function $w_{T}(t)$ is utilized with a rectangular shape. $\mathrm{N}_{\mathrm{ST}}$ is the total number of subcarriers. $\Delta f$ is the sub-carrier spacing while $T_{G I}$ means the guard interval time for creating the cyclic prefix. $r(t)$ is the concatenation of these SC-FDMA symbols, which is described as [18] 


$$
r(t)=\sum_{n=-\infty}^{\infty} w_{T}\left(t-n T_{S}\right)\left[\sum_{k=-\frac{N_{S T}}{2}}^{N_{S T} / 2} a_{k, n} e^{j 2 \pi k \Delta f\left(t-T_{G I}-n T_{S}\right)}\right]
$$

where $T_{s}$ is the symbol duration time. The transmitted SC-FDMA signal $s(t)$ then is written as [13]

$$
s(t)=\operatorname{Re}\left[r(t) e^{j 2 \pi f_{c} t}\right]
$$

where $\operatorname{Re}[*]$ is the real part of the signal while $f_{c}$ denotes the carrier frequency of the LTE system. The parameters used in the math model for testing the LTE signal are listed in Table 2.3 [18].

\begin{tabular}{|l|l|}
\hline SC-FDMA parameter & LTE \\
\hline Subcarrier frequency spacing $\Delta f$ & $15 \mathrm{k} \mathrm{Hz}$ \\
\hline Number of used subcarriers $N_{S T}$ & 1200 \\
\hline FFT size & 8192 \\
\hline Total symbol time $T_{S}$ & $71.36 \mu \mathrm{s}$ \\
\hline Data modulation & $16-\mathrm{QAM}$ \\
\hline Sampling frequency $1 / T_{S}$ & $15.36 \mathrm{MHz}$ \\
\hline Circular prefix $T_{G I}$ & $4.69 \mu \mathrm{s}$ \\
\hline
\end{tabular}

Table 2.3 Parameters applied in the simulation math model

The general formula of the power spectral density (PSD) of a SC-FDMA baseband signal is written as [4]

$$
\mathrm{P}_{r}(f)=\frac{R_{S}}{4} \sum_{\mathrm{k}=-\frac{\mathrm{N}_{\mathrm{ST}}}{2}}^{\mathrm{N}_{\mathrm{ST}} / 2}\left[|W(f-k \Delta f)|^{2}+|W(-f-k \Delta f)|^{2}\right]
$$


where $R_{S}=\frac{1}{T_{S}}$ is the symbol rate and $W(f)$ defines the Fourier transform of the rectangular window function in this case. Figure 2.9 illustrates that the simulated and measured baseband signal spectra achieve good matches with the spectrum of the calculated expression (2.14) [19].

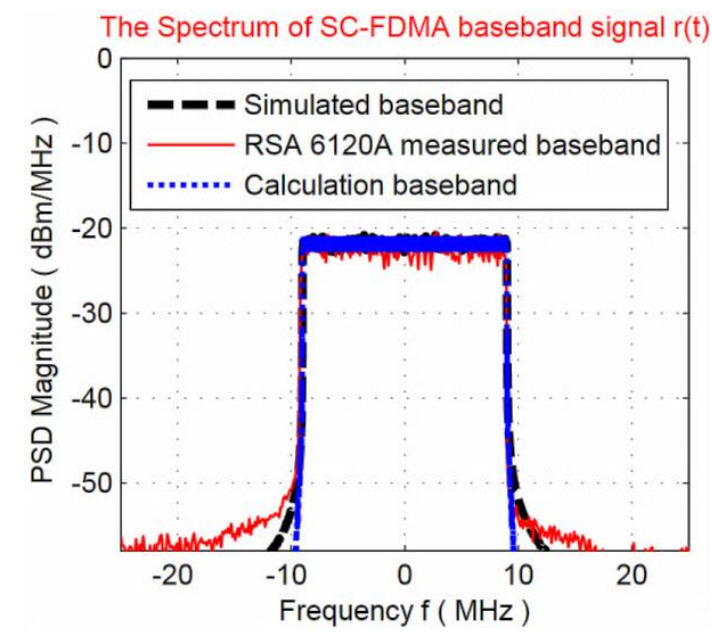

Figure 2.9 Power spectrum comparison among simulation, measurement and calculation results of baseband SC-FDMA signal

\subsection{Spectrum Regrowth of Amplified LTE Uplink Signals}

From expression (2.13), a RF power amplifier has the analytical model shown as

$$
y(t)=O\{s(t)\}=\mathrm{F}[r(t)] \cos \left(2 \pi f_{c} t+\varphi[r(t)]\right)
$$

where $O\{*\}$ is the operation of amplifier while $\mathrm{F}[*]$ and $\varphi[*]$ are $\mathrm{AM} / \mathrm{AM}$ and $\mathrm{AM} / \mathrm{PM}$ conversions respectively, depending on the model characteristics of the amplifier. 
Implementing an autocorrelation of $r(t)$ and by the corollary to the WienerKhintchine Theorem [12], the final power spectrum $P_{y}(f)$ of y(t) will become:

$$
P_{y}(f)=A_{1} \frac{P_{1}\left(f-f_{c}\right)}{N_{S T}}+A_{2} \frac{P_{3}\left(f-f_{c}\right)}{N_{S T}{ }^{3}}
$$

$P_{1}$ represents the PSD of a SC-FDMA baseband signal and $P_{3}$ is equivalent to the convolution results of three $P_{1}$ values. $A_{1}$ and $\mathrm{A}_{2}$ are two factors having values after calculation shown below:

$$
\begin{gathered}
A_{1}=2 P_{0}-12 P_{0}^{2} 10^{-\frac{I P_{3}}{10}}+18 P_{0}^{3} 10^{-\frac{I P_{3}}{5}} \\
A_{2}=48 P_{0}^{3} 10^{-\frac{I P_{3}}{5}}
\end{gathered}
$$

where $P_{0}=\frac{a_{1}^{2} N_{S T}}{4}$ is the linear output power of the amplifier.

Another form in terms of $I P_{3}$, evolved from the power spectrum result of $P_{y}(f)$ in (2.16) and (2.17), is described as:

$$
D_{1} \times 10^{-\frac{I P_{3}}{5}}+D_{2} \times 10^{-\frac{I P_{3}}{10}}+D_{C}=0
$$

where

$$
\begin{gathered}
D_{1}=\frac{18 P_{0}^{3}}{N_{S T}{ }^{3}} P_{1}\left(f-f_{c}\right)+\frac{48 P_{0}^{3}}{N_{S T}{ }^{3}} P_{3}\left(f-f_{c}\right) \\
D_{2}=-\frac{12 P_{0}^{2}}{N_{S T}{ }^{3}} P_{1}\left(f-f_{c}\right) \\
D_{C}=\frac{2 P_{0}}{N_{S T}} P_{1}\left(f-f_{c}\right)-P_{y}(f)
\end{gathered}
$$

In order to get the explicit expression of $I P_{3}$, the form in (2.18) is solved with (2.19):

$$
I P_{3}=-10 \times \log _{10}\left(\frac{-D_{2}+\sqrt{D_{2}^{2}-4 D_{1} D_{C}}}{2 D_{1}}\right)
$$


According to the theoretical expression, the value of $I P_{3}$ depends on a particular outof-band emission power level. The emission level of the power spectrum in a given band from $f_{1}$ and $f_{2}$ is described as $P_{I M_{3}}\left(f_{1}, f_{2}\right)$ [13]:

$$
P_{I M_{3}}\left(f_{1}, f_{2}\right)=\int_{f_{1}}^{f_{2}} P_{y}(f) d f
$$

From the integration of $P_{y}(f)$, another group of coefficients $D_{1}^{\prime}, D_{2}^{\prime}$ and $D_{C}^{\prime}$ is calculated as [13]

$$
\begin{gathered}
D_{1}^{\prime}=\frac{18 P_{0}^{3}}{N_{S T}^{3}} \int_{f_{1}}^{f_{2}} P_{1}\left(f-f_{c}\right) d f+\frac{48 P_{0}^{3}}{N_{S T}^{3}} \int_{f_{1}}^{f_{2}} P_{3}\left(f-f_{c}\right) d f \\
D_{2}^{\prime}=-\frac{12 P_{0}^{2}}{N_{S T}{ }^{3}} \int_{f_{1}}^{f_{2}} P_{1}\left(f-f_{c}\right) d f \\
D_{C}^{\prime}=\frac{2 P_{0}}{N_{S T}} \int_{f_{1}}^{f_{2}} P_{1}\left(f-f_{c}\right) d f-P_{I M_{3}}\left(f_{1}, f_{2}\right)
\end{gathered}
$$

However, the new expression of $I P_{3}$ is of the same form with (2.20) except the latter coefficients substitute for the previous ones respectively, giving a clear view of state transition between the out-of-band emission power level and $I P_{3}$. 


\section{Chapter 3 The Digital Pre-Distortion of the SC-FDMA Signals in LTE Up-link}

\section{System}

RF power amplifiers (PA) bring nonlinear distortion that degrades signal transmission quality and increases inter-channel interference [20]. In addition, with the development of fourth generation (4G) mobile communication systems, the modern wideband signal has aroused the attention to the issues wherein the accuracy of PA modelling has been influenced by memory effects, which is always not considered in other narrowband applications [21]. This new issue arises a request for applying DPD algorithms with the memory effect to the wideband LTE systems since traditional predistorters always perform poor accuracy with memory-less models. A DPD algorithm is applied to the SC-FDMA signals in the uplink of the LTE systems. It captures the nonlinearity and memory effects with two separate models based on the Hammerstein structure first, and then applies the inverse polynomial model and the inverse IMARMA model to compensate previous two parts of effect respectively. The improvement of the DPD algorithm for the SC-FDMA signals was verified with a series of experimental measurements.

\subsection{Pre-Distortion Review}

The digital pre-distorter (DPD) is widely applied by employing digital baseband distortion ahead of the amplifier to compensate for the nonlinearity effects and provide efficiency enhancements for high power amplifiers in the different communication systems. This thesis presents a digital pre-distorter first applied to linearize SC-FDMA 
signals having the PA distortion with memory effect using an efficient and flexible inverse intermodulation and Autoregressive Moving-Average (IM-ARMA) model in the LTE uplink system.

\subsection{Spectrum Regrowth Analysis with Pre-Distortion}

The DPD algorithm is divided into two parts shown in Figure 3.1 [4]:

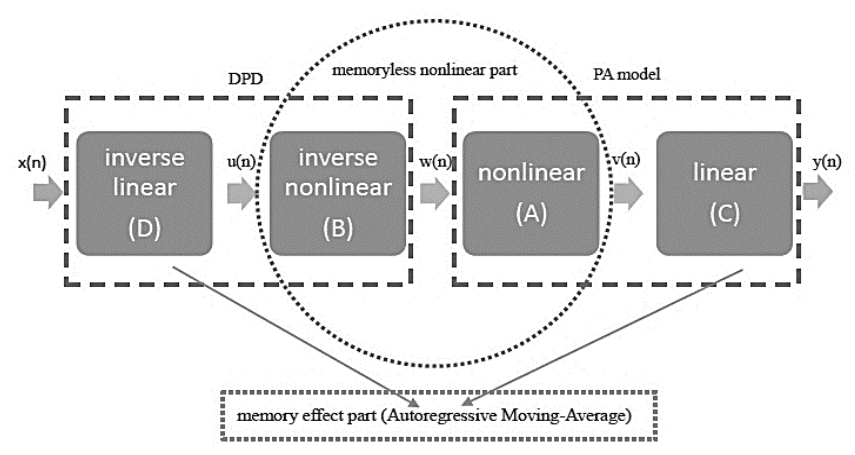

Figure 3.1 The Structure of DPD process

The PA is modelled by two blocks: a memory-less nonlinear block (A) and a LTI (linear time invariant) block (C) presenting memory effect; The digital pre-distorter is modelled by two inverse functions according to PA model: inverse nonlinear block (B) and an inverse LTI block (D). The inverse nonlinear block (B) compensates the memory-less distortion of the PA modelled by block (A), and the inverse LTI block (D) cancels the memory effect presented by block (C) [4]. The description of each block will be discussed below. 


\subsubsection{Block A: The Nonlinear Model}

The Taylor series model $\mathrm{v}(\mathrm{n})$ of a high power amplifier is applied for the polynomial model, expressed as [22]:

$$
\mathrm{v}(\mathrm{n})=a_{1} \mathrm{w}(n)+a_{3} \mathrm{w}^{3}(n)+\cdots+a_{M} w^{M}(n)
$$

where $\mathrm{w}(n)$ represents the input. The coefficient series $a_{m}$ is determined by the linear gain $G$ of the amplifier and $m$-order intercept point $\left(I P_{m}\right)$. It should be noticed that only terms with odd-order features are included in this model due to the simple filtration process with even-order $I P_{m}$ terms, which are always kept far away from the center of passband. Moreover, the coefficient series $a_{m}$ have the form of an exponential function. Accordingly, following function is proposed to describe the relationship between $a_{m}$ and PA intercept points [4]:

$$
a_{m}=-\frac{2^{\frac{m-1}{2}}}{\left(\frac{m+1}{2}\right)} 10^{\left(\frac{I M P_{m}-m P_{t}+m G}{20}\right)}
$$

where $\left(\frac{m}{\frac{m+1}{2}}\right)$ gives the number of different combinations of $\frac{m+1}{2}$ elements that can be chosen from an $m$-element set. $G$ is the gain of the PA while $I M P_{m}$ stands for the $m^{\text {th }}$ order intermodulation product, which is the difference between the original output signal and the $m^{\text {th }}$ order intermodulation output power from a simple two tone test.

\subsubsection{Block C: The Memory Effect Model}

With the development of fourth generation mobile communication system, the modern wideband signal has aroused the attention to the issues wherein the accuracy of PA 
modelling has been influenced by memory effects which is always not considered in narrowband applications.

For the memory effect part, the output is determined by both current and past inputs [4]. Therefore, the time delays of both inputs and outputs are required to be involved in the algorithm. Due to the linear delay property of the memory effect, the Autoregressive Moving-Average (ARMA) model is used as:

$$
\sum_{k=0}^{K} p(k) y(n-k)=\sum_{l=0}^{L} q(l) v(n-l)
$$

where $v$ and $y$ mean the input and output functions, while $k$ and $l$ are two delays of outputs and inputs respectively. $p(k)$ and $q(l)$ are coefficients of AR and MA parts of the model. The transfer function is presented as [4]:

$$
H(z)=\frac{\sum_{k=0}^{K} q(k) z^{-k}}{\sum_{l=0}^{L} p(l) z^{-l}}
$$

The advantages of the ARMA filter contain: It is quite simple to obtain the coefficients from several mature algorithms [22], also the memory effect can be reduced easily by applying the pre-distortion algorithm which is the inverse transfer function of the ARMA filter.

Finally the PA model with memory effect is described with nonlinearity polynomial function and memory effect function [4]:

$$
\sum_{k=0}^{K} q(k) y(n-k)=\sum_{l=0}^{L} p(l)\left[a_{1} w(n-l)+a_{3} w^{3}(n-l)+\cdots+a_{M} w^{M}(n-l)\right]
$$




\subsubsection{Block B: The Inverse Polynomial of PA}

To pre-distort the PA, we start from the linearization of the memory-less distortion modelled in (3.1) for the block A. A developed formula based on the polynomial model is described as [4]:

$$
w(t)=b_{1} u(t)+b_{3} u^{3}(t)+\cdots+b_{P} u^{P}(t)
$$

By replacing $w(t)$ in (3.6), the whole system in (3.1) then can be expressed as:

$$
v(t)=h_{1} u(t)+h_{3} u^{3}(t)+\cdots+h_{M \cdot P} u^{M \cdot P}(t)
$$

The $p^{\text {th }}$ order nonlinearity requests at least the same order polynomial pre-distorter for linearization. From the definition of $p^{\text {th }}$ order inverse method [22], the coefficients are described as:

$$
h_{1}=a_{1}, h_{3}=0, \ldots h_{p}=0
$$

PA will have no nonlinearity output with $p^{\text {th }}$ of pre-distorter polynomial order if all the equations in (3.8) are satisfied. To achieve the goal, the series of coefficient $b_{P}$ is calculated as [4]:

$$
b_{p}=-\sum_{\substack{m=3 \\
m \text { is odd integer }}}^{M}\left[\left(\begin{array}{cc}
\sum_{\substack{\sum_{k=1}^{m} i_{k}=p \\
\sum_{k=1}^{m} i_{k}=m \\
i_{k} \text { is odd integer }}}^{b_{i_{1}} b_{i_{2}}} \ldots b_{i_{m}}
\end{array}\right) \frac{a_{m}}{a_{1}}\right]
$$

where $\mathrm{M}$ is the maximum order within the PA model.

\subsubsection{Block D: The Inverse Memory Effect of PA}

Since linear signals $v(t)$ has been generated by counteracting the effect from both the inverse polynomial block (B) and the polynomial block (A), the memory effect is necessary to be removed then. The memory effect (LTI block C) is modelled as 
ARMA in (3.5), so the inverse block (D) should have the same ARMA structure with inverse AR and MA coefficients. This was achieved by exchanging the positions of pole and zero. The output $u(n)$ of inverse ARMA block (D) and the inverse transfer function can be indicated as:

$$
\begin{gathered}
\sum_{k=0}^{K} p(k) y(n-k)=\sum_{l=0}^{L} q(l) v(n-l) \\
H(z)=\frac{\sum_{k=0}^{K} q(k) z^{-k}}{\sum_{l=0}^{L} p(l) z^{-l}}
\end{gathered}
$$




\section{Chapter 4 Analysis and Experimental Study of SC-FDMA Signals}

\subsection{SC-FDMA Spectrum for LTE Up-link System}

The simulation section followed a similar sequence to the transmitter structure in Figure 2.7. The simulation process is shown in Figure 4.1. 16-QAM modulated complex symbols were applied to the SC-FDMA system. The normalization factor KMOD was chosen as $\frac{1}{\sqrt{10}}$ to achieve the same average power for mappings in this case. Compared with the OFDM system, the extra N-point DFT block transferred the system into a single-carrier one which avoids a high PAPR and improves system throughput by confining the power within a certain threshold. Then during the subcarrier mapping, the Localized FDMA method was chosen through using a certain fraction of the whole bandwidth. The amplifier simulation was then built with the Taylor model. For the calculation section, a rectangular window function for the SCFDMA signal was applied prior to calculating the PSD of the RF signal. Then the total bandwidth was split in two sets of parts shown in expression (2.13) and (2.14), and the whole analytical models were shown and discussed in previous sections. 


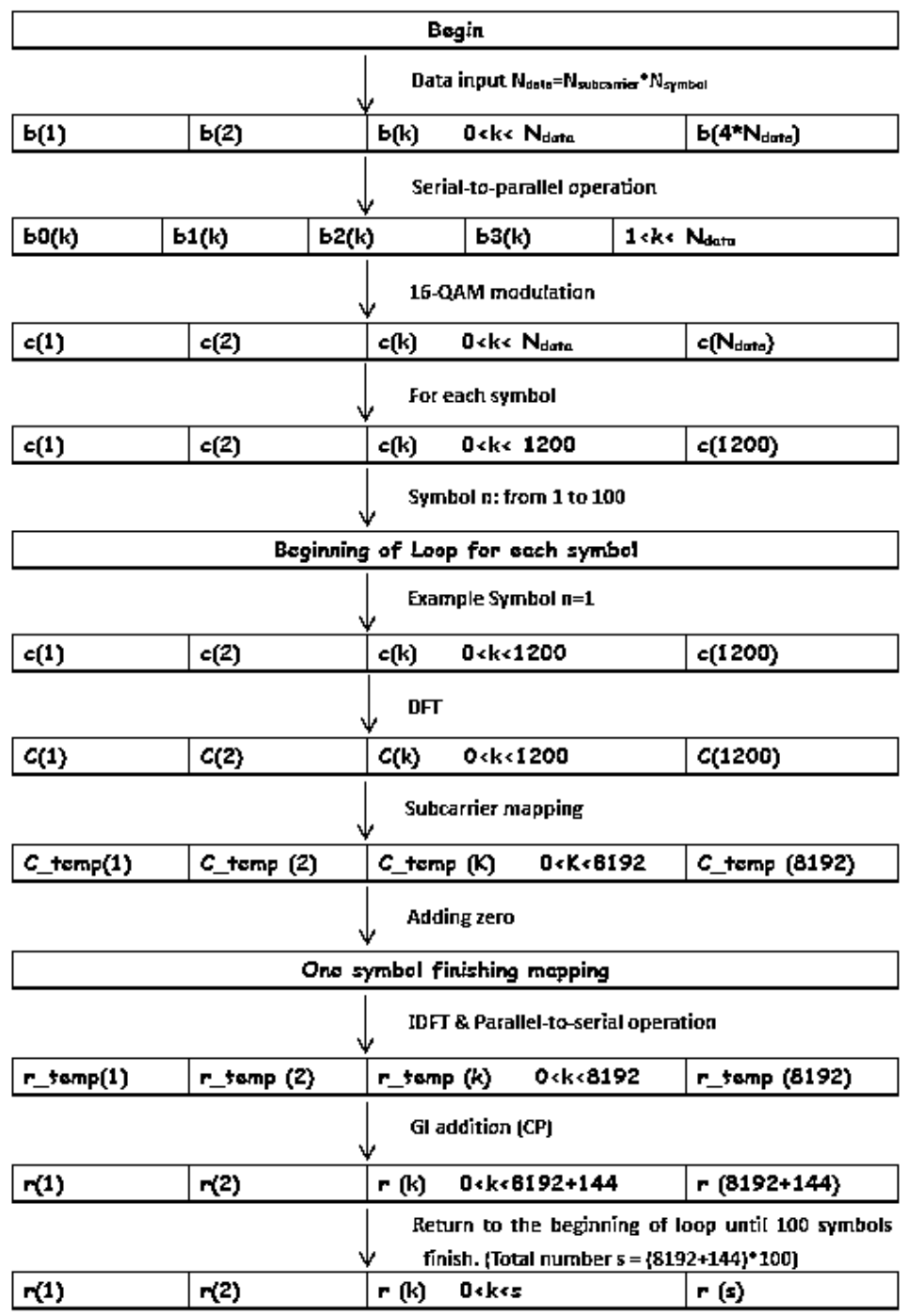

Figure 4.1 The flow chat of simulation process

Finally, the measurement was made with the instruments in Figure 4.2. The Model types and descriptions are included in Table 4.1. The LTE signal was generated by Agilent E4438C ESG vector signal generator using a carrier frequency of $1 \mathrm{GHz}$, and power level set as $-10 \mathrm{dBm}$. The sampling frequency was $122.88 \mathrm{MHz}$ with 801 points acquired and analyzed for outputs. The analyzing span was set as $100.0 \mathrm{MHz}$ and 
reference level as $-2.00 \mathrm{dBm}$. The ZFL-1000LN RF power amplifier was placed between the generator and the Tektronix RSA 6120A real-time spectrum analyser. It had a $13 \mathrm{dBm} I P_{3}$ and an output gain of $20 \mathrm{~dB}$ according to the datasheet. The output spectrum of the amplified SC-FDMA signal from the spectrum analyzer was then reconstructed by Matlab R2012b.

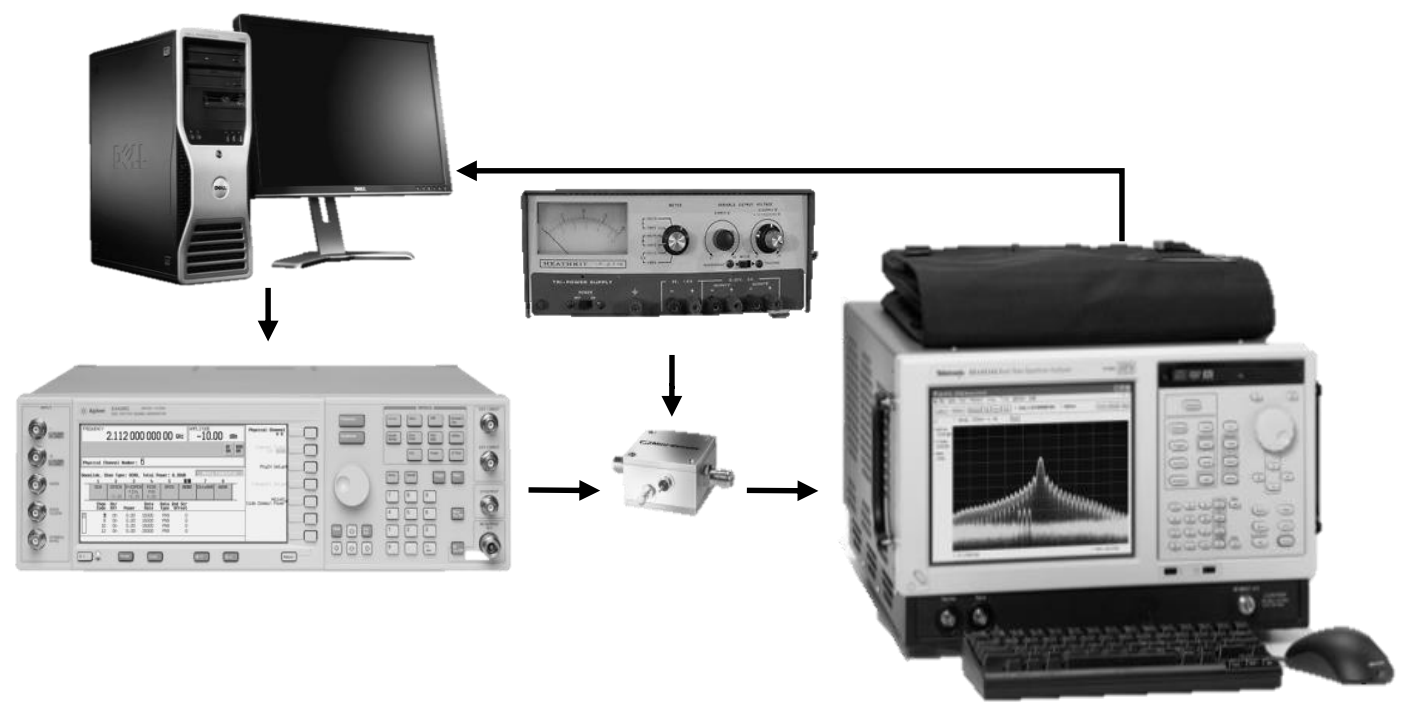

Figure 4.2 Experimental setup of spectrum measurement of SC-FDMA signals

\begin{tabular}{|l|l|l|}
\hline & Description & Model No. \\
\hline Agilent & Signal Generator & E4438C \\
\hline Mini-Circuits & RF Power Amplifier & ZFL-1000LN+ \\
\hline Heathkit & Power Supply & IP 2718 \\
\hline Tektronix & Spectrum Analyzer & RSA6120A \\
\hline
\end{tabular}

Table 4.1 Experimental Instruments 


\subsubsection{SC-FDMA Spectrum with $I P_{3}$ only}

Figure 4.3 shows that the final simulated and measured RF amplifier spectra have high similarity to the calculated spectrum from (2.15) in both the pass-band and the shoulder area.

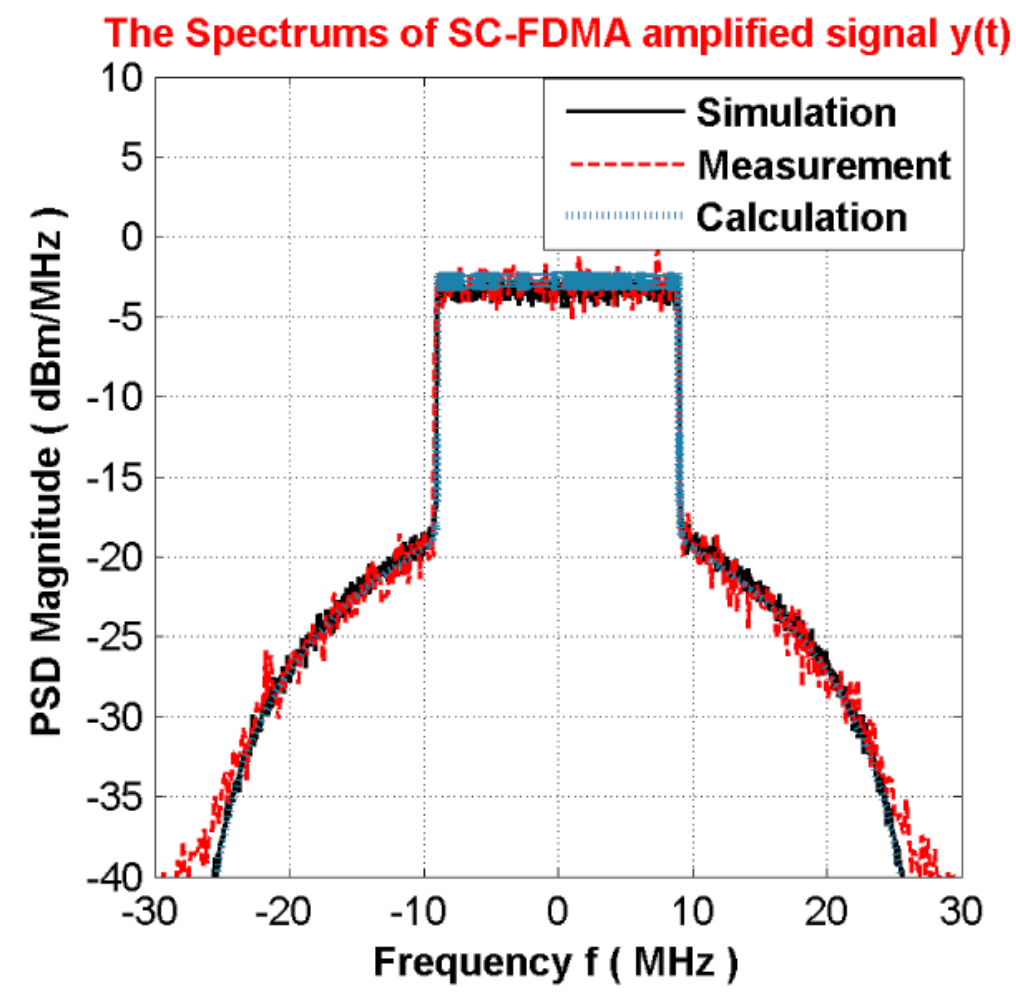

Figure 4.3 Power spectrum comparison among amplified signals with $I P_{3}$ only in up-

link LTE Wireless system

From Figure 4.3, the bandwidth B is observed as about $10 \mathrm{MHz}$. For $|f|<\mathrm{B}$, where $f$ equals the frequency in the horizontal axis in the figure, this part is known as the passband containing the linear output power density for an ideal power amplifier performance. For the out-of band range, with the frequency range approximately from 
10 to $30 \mathrm{MHz}$ shown in Figure 4.3, the PSD output $P_{y}(f)$ is mainly related to 3rd intermodulation since only $I P_{3}$ is considered in the calculation. The result shows that the out-of-band spectrum density is always controlled by intermodulation, and these out-of-band frequency components are usually named as spectrum regrowth and include the most significant part of the emission of a power amplifier.

Furthermore, for the band $|f|>3 \mathrm{~B}$, which is about $30 \mathrm{MHz}$ shown in Figure 4.3, the power density is still not zero because there always exists some noise, which cannot be neglected in terms of power level compared with the output spectrum. This point of view is not proposed in Figure 4.3 as the result of some limitations. Firstly, only $I P_{3}$ is considered in this part. From the figure, it can be concluded that it has great difficulty distinguishing $I P_{3}$ with ambient noise since the power level of higher-order intermodulation points is not considerable in this part, which is more than $40 \mathrm{~dB}$ lower than the amplified output power. Secondly, because the datasheet of a power amplifier does not often include the value of $I P_{5}$ or higher orders, it is not reliable to simulate and calculate the amplified spectrum with higher order intermodulation at this point.

\subsubsection{SC-FDMA Spectrum with both $I P_{3}$ and $I P_{5}$}

From the Figure 4.4, the bandwidth B is modified to about 4.5 MHz. In this case, The PSD outputs of simulated and measured signal spectrums achieve good matching with the spectrum of calculated expression for the band $|f|<3 \mathrm{~B}$. For the band $|f|>3 \mathrm{~B}$, which is about $13.5 \mathrm{MHz}$ shown in Figure 4.4, The extra noise has a relatively deep influence on the output spectrum with fifth order intercept points in terms of power 
level. Furthermore, as referred before, the value of $I P_{5}$ is measured and calculated by two-tone test with (2.5), so some errors may be generated since the amplifier is not ideal.

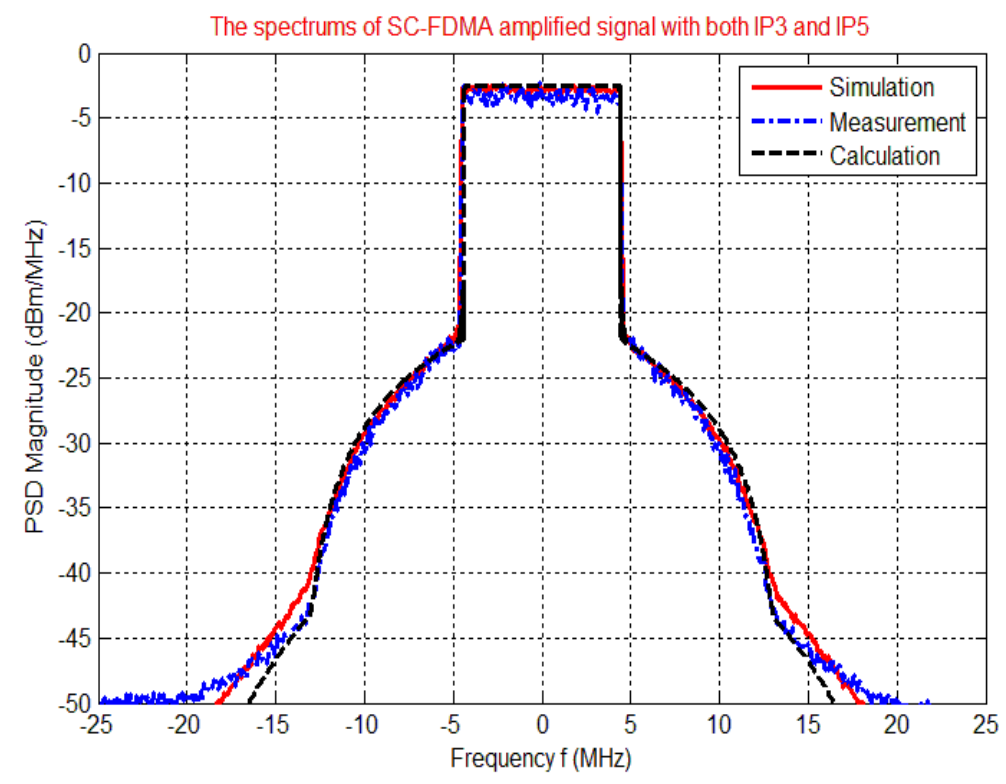

Figure 4.4 Power spectrum comparison with both $I P_{3}$ and $I P_{5}$

\subsection{The Digital Pre-Distortion of the SC-FDMA Signals}

The same setup of instruments shown in Figure 4.2 and Table 4.1 was applied to verification of the DPD algorithm. The SC-FDMA signal was generated by the Agilent E4438C ESG vector signal generator using a carrier frequency of $1 \mathrm{GHz}$, and power level $-17 \mathrm{dBm}$. The bandwidth of the signals is $10 \mathrm{MHz}$. A Tektronix RSA $6120 \mathrm{~A}$ real-time spectrum analyser was used to measure the original and the amplified SC-FDMA signals through the ZFL-1000LN RF power amplifier produced by Mini - 
Circuit. The output gain is $20 \mathrm{~dB}$ according to the datasheet. The DPD is finally implemented by MATLAB R2010b.

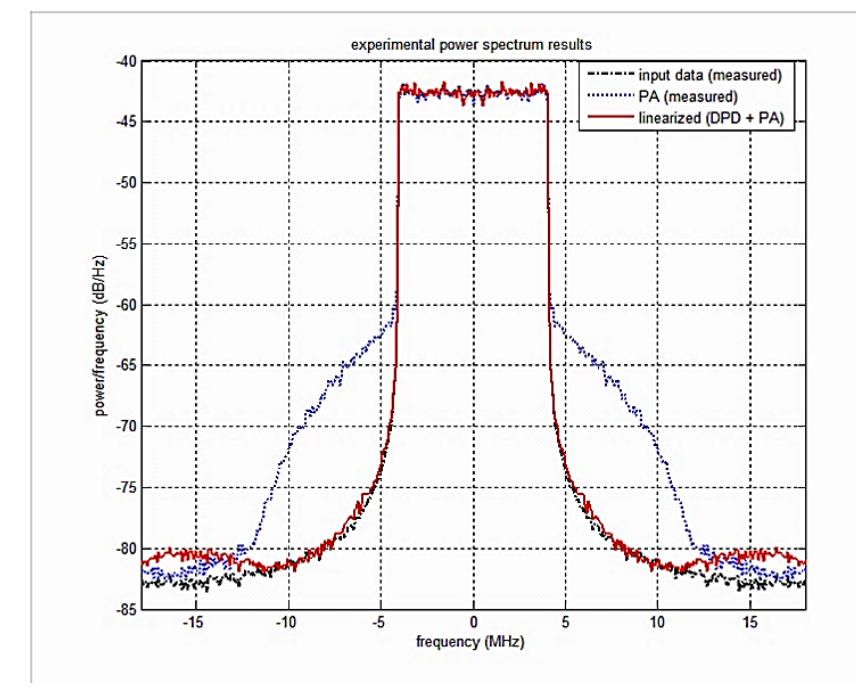

Figure 4.5 The power spectrum comparison of SC-FDMA signals

Figure 4.5 shows the power spectrum of the original input signal without amplification, the amplified signal without DPD, and the amplified signal with DPD. The order of the nonlinear model we used is up to 5 and the memory depth is 2 for the AR part and 2 for the MA part in the block C and block D. With the DPD algorithm, the amplified power spectrum is very similar to the original input spectrum after the normalization. The PAPR (Adjacent channel power ratio) is improved with $12 \mathrm{~dB}$ achieved in the area close to the fundamental frequency. The performance in the further area (offset > $|12 \mathrm{~dB}|)$ is a little worse because the inverse polynomial could force the signals to have no nonlinearity up to the $\mathrm{p}^{\text {th }}$ order of the pre-distorter polynomial, regardless of the PA's polynomial order. Certain nonlinear terms of orders higher than $p$ may still exist 
in the compensated output. However, those interferences could be filtered out easily since they are relatively far from the fundamental frequency. 


\section{Chapter 5 Concluding Remarks}

The research results confirm that spectrum regrowth with RF power amplifiers has been specified and measured efficiently. This sort of design includes the advantages of SC-FDMA and is of great help for both designing RF power amplifiers and for further improving spectrum regrowth with pre-distortion outside channels, which can be extended to other wireless systems based on SC-FDMA modulation. The spectrum regrowth analysis technology of SC-FDMA signals is applied and verified in the testing and simulation work with LTE signals. From the experimental result, it was supposed that the fifth or higher order intermodulation has little effect on out-of-band emission power levels. The theoretical result from reasonable calculation is verified along with experiment outputs. The relationship between power spectrum levels and nonlinearity coefficients of RF power amplifiers, mainly $I P_{3}$ in this thesis, is verified in the analytical model. Also the ARMA based DPD algorithm with memory polynomial model is applied to SC-FDMA signals in the LTE uplink system. The experimental results showed that the DPD algorithm reduced the spectrum regrowth by $12 \mathrm{~dB}$. With the expression of amplified power emission levels we derived, the result may help meet the out-of-band requirements of the LTE standards and make progress with digital pre-distortion in the up-link of LTE systems. 


\section{References}

[01]. H. Xiao, "Spectrum Modeling for Linear RF Power Amplifier Design for Digital Cellular Communication Signals”, Ph.D. dissertation, Portland State University, 1999.

[02]. C. M. Liu, "Spectral Modeling and Nonlinear Distortion Analysis of OFDM Based Wireless LAN Signals”, Ph.D. dissertation, Portland State University, 2005.

[03]. B. S. Chen, "Spectrum Regrowth for OFDM-Based LTE and WINAX Systems", M.S. thesis, Portland State University, 2012.

[04]. X. Li, "Nonlinearity Analysis and Predistortion of $4 G$ Wireless Communication Systems”, Ph.D. dissertation, Portland State University, 2013.

[05]. F. S. Al-kamali, M. I. Dessouky, B. M. Sallam, F. Shawki, F. E. Abd El-Samie, "Impact of the Power Amplifier on the Performance of the Single Carrier Frequency Division Multiple Access System”, Telecommunication Systems, vol. 52, no. 1, pp 31-38, 2013.

[06]. R. Sobot, “Wireless Communication Electronics”, Springer Science+Busness Media, LLC, 2012.

[07]. P. Jantunen, "Modelling of Nonlinear Power Amplifiers for Wireless Communications”, M.S. thesis, Helsinki University of Technology, 2004.

[08]. S. C. Cripps, “RF Power Amplifiers for Wireless Communications”, Artech House, INC, 1999.

[09]. L. W.Couch, "Digital and Analog Communication Systems", New Jersey: Prentice-Hall, Inc., 1996. 
[10]. W. Kester, "Intermodulation Distortion Considerations for ADCs", Analog Devices, Tutorial MT-012, Rev. A, 2009.

[11]. H. Xiao, Q. Wu, F. Li, “Measure a power amplifier's fifth-order intercept point”, RF Design, April, pp. 54-56, 1999.

[12]. X. Li, B.S. Chen, C.M. Liu, X.R. Wang, K.R. Cho, F. Li, “Spectrum modelling and regrowth for 4G wireless signals", Electronics Letters, Vol. 48, No 4, 2012.

[13]. C.M. Liu, F. Li, "Nonlinearity analysis of OFDM-based wireless systems in Orthogonal frequency division multiple access fundamentals and applications", Auerbach Publications, Orthogonal Frequency Division Multiple Access Fundamentals and Applications, Chapter 3, pp. 41-65, 2010.

[14]. M. Jamal, B. Horia, K. Maria, I. Alexandru, "Study of Multiple Access Schemes in 3GPP LTE OFDMA vs. SC-FDMA", IEEE Applied Electronics, International Conference, pp. 1-4, 2011.

[15]. Xixia Leader in Converged IP Testing, "SC-FDMA Single Carrier FDMA in LTE”, IXIA, 915-2725-01 Rev A, 2009.

[16]. M. Rumney, "3GPP LTE: Introducing Single-Carrier FDMA", Agilent Measurement Journal, vol. 4, pp. 18-27, 2008.

[17]. H.G. Myung, J. Lim, D.J. Goodman, "Single Carrier FDMA for Up-link Wireless Transmission", IEEE Transactions on Vehicular Technology Magazine, vol. 3, no. 1, 2006.

[18]. S. Parkvall, "TS 36.211-11.2.0 E-UTRA Physical channels and modulation", The 3rd Generation Partnership Project, Rel. 11, Version 11.2.0, 2013. 
[19]. C.W. Zhu, X. Li, F. Li, 'Nonlinear analysis of SC-FDMA spectrum for LTE up-link' International Journal of Electronics Letters, Vol. 2, Issue 1, pp. 30-36, 2014.

[20]. H. Gandhi, "A Flexible Volterra-Based Adaptive Digital PreDistortion Solution for Wideband RF Power Amplifier Linearization", Microwaves and RF Magazine, Jul. \& Aug. 2008.

[21]. Aug. 2008.X. Li, F. Li, "RF Power Amplifiers Nonlinear Modeling with Memory Effect", International Journal of Electronics Letters, vol. 1, no. 1, pp. 4449, 2013.

[22]. M. Schetzen, "Theory of $p^{\text {th }}$-order inverses of nonlinear systems", Circuits and Systems, IEEE Transactions, Vol. 23, Issue 5, 2003. 